\title{
Jiasongfangiella alkanivorans gen. nov., sp. nov., a novel bacterium with degradation of alkanes isolated from the sediment of the Mariana Trench
}

\author{
YULI WEI ${ }^{1}$, JIAHUA WANG ${ }^{2}$, YAN ZHANG ${ }^{3}$ AND \\ JIASONG FANG ${ }^{1}$ \\ ${ }^{1}$ Shanghai Ocean University \\ ${ }^{2}$ College of Marine Sciences, Shanghai Ocean University \\ ${ }^{3}$ Shanghai Ocean Univeristy \\ Presenting Author: yl_wei@shou.edu.cn
}

It was reported that a bundant $n$-alkanes were observed in sinking particles at 2000, 4000, and $6000 \mathrm{~m}$ seawater of the Mariana Trench. However, the active hydrocabonclastic microorganisms in trench sediment are largely unknown. A Gram-staining-negative, facultative anaerobic, long-rod shaped with flagellum bacterium designated strain $\mathrm{C} 2-1$, was isolated from the sediment at the depth of $3000 \mathrm{~m}$ in the Mariana Trench. Based on GTDB taxonomy, strain C2-1 is considered to represent the uncultured genus "UBA2009", most closely related to the genus Thalassolituus and Oliebacter. The complete genome of strain $\mathrm{C} 2-1$ is $4.39 \mathrm{Mb}$ with $53.1 \% \mathrm{G}+\mathrm{C}$ content and encodes 3,842 genes, including one $n$-alkane hydroxylase alkB and several genes involved in carbohydrate utilization. Physiological experiments confirmed under both normal and in situ hydrostatic pressure (30 Mpa), strain C2-1 could utilize straight-chain $n$-alkanes (C16-C18) as sole carbon source that was detected from our in situ samples, for which the name Jiasongfangiella alkanivorans is proposed. Environmentally, 18 metagenome-assembled genomes (MAGs) of "Jiasongfangiella" were retrieved from public available datasets sampled from seawater and underground water, indicating wide distribution and various ecotypes of this genus. Compared to 16 genomes from 6 most closely related genus, strain C2-1 and most of those MAGs occupy genus-specific nitrate reductase narGHI, which might contribute to the adaption of low oxygen environment in deep marine sediments. Taken together, strain C2-1 representing as a novel facultative $n$-alkane degrader provides a good model to investigate organic carbon degradation in the deep-sea ecosystems, which may facilitate a deeper understanding of microbial environmental adaptability and better utilization for bioremediation purposes in the future. 